\title{
COVID-19 Vaccination Dynamics in the US: Coverage Velocity and Carrying Capacity Based on Socio-demographic Vulnerability Indices in California
}

\author{
Alexander Aram Bruckhaus ${ }^{1}$ - Aidin Abedi ${ }^{2}$. Sana Salehi ${ }^{1} \cdot$ Trevor A. Pickering $^{3} \cdot$ Yujia Zhang $^{1} \cdot$ Aubrey Martinez $^{1}$. \\ Matthew Lai ${ }^{1} \cdot$ Rachael Garner ${ }^{1}$. Dominique Duncan ${ }^{1}$
}

Accepted: 1 November 2021 / Published online: 19 November 2021

(c) The Author(s), under exclusive licence to Springer Science+Business Media, LLC, part of Springer Nature 2021

\begin{abstract}
Coronavirus disease 2019 (COVID-19) disparities among vulnerable populations are of paramount concern that extend to vaccine administration. With recent uptick in infection rates, dominance of the delta variant, and authorization of a third booster shot, understanding the population-level vaccine coverage dynamics and underlying sociodemographic factors is critical for achieving equity in public health outcomes. This study aimed to characterize the scope of vaccine inequity in California counties through modeling the trends of vaccination using the Social Vulnerability Index (SVI). Overall SVI, its four themes, and 9228 data points of daily vaccination numbers from December 15, 2020, to May 23, 2021, across all 58 California counties were used to model the growth velocity and anticipated maximum proportion of population vaccinated, defined as having received at least one dose of vaccine. Based on the overall SVI, the vaccination coverage velocity was lower in counties in the high vulnerability category $(\mathrm{v}=0.0346,95 \%$ CI $0.0334,0.0358)$ compared to moderate $(\mathrm{v}=0.0396,95 \%$ CI $0.0385,0.0408)$ and low $(v=0.0414,95 \%$ CI $0.0403,0.0425)$ vulnerability categories. SVI Theme 3 (minority status and language) yielded the largest disparity in coverage velocity between low and high-vulnerable counties $(\mathrm{v}=0.0423$ versus $\mathrm{v}=0.035, P<0.001)$. Based on the current trajectory, while counties in low-vulnerability category of overall SVI are estimated to achieve a higher proportion of vaccinated individuals, our models yielded a higher asymptotic maximum for highly vulnerable counties of Theme $3(\mathrm{~K}=0.544,95 \%$ CI $0.527,0.561)$ compared to low-vulnerability counterparts $(\mathrm{K}=0.441$, $95 \%$ CI $0.432,0.450)$. The largest disparity in asymptotic proportion vaccinated between the low and high-vulnerability categories was observed in Theme 2 describing the household composition and disability ( $\mathrm{K}=0.602$, 95\% CI 0.592, 0.612; versus $\mathrm{K}=0.425,95 \% \mathrm{CI} 0.413,0.436$ ). Overall, the large initial disparities in vaccination rates by SVI status attenuated over time, particularly based on Theme 3 status which yielded a large decrease in cumulative vaccination rate ratio of low to high-vulnerability categories from 1.42 to $0.95(\mathrm{P}=0.002)$. This study provides insight into the problem of COVID-19 vaccine disparity across California which can help promote equity during the current pandemic and guide the allocation of future vaccines such as COVID-19 booster shots.
\end{abstract}

Keywords COVID-19 $\cdot$ Vaccination $\cdot$ Resource allocation $\cdot$ Health equity $\cdot$ Minorities

\section{Introduction}

Alexander Aram Bruckhaus and Aidin Abedi have contributed equally as co-first authors.

Sana Salehi

ssalehi@usc.edu

Extended author information available on the last page of the article
The coronavirus disease 2019 (COVID-19) has disproportionately impacted minority groups in terms of the number of cases and mortality rates, which further warrants equitable vaccine rollout to assist the communities in dire need of medical support [1]. Data from a Centers for Disease Control and Prevention (CDC) report revealed that in most U.S. states, vaccination coverage was higher in low- and moderate-vulnerability counties compared to high-vulnerability counterparts [2]. A similar report revealed that as of May 24, 
2021, the percentage of White people who had received at least one dose of COVID-19 vaccine was about 1.5 and 1.3 times higher than Black and Hispanic people, respectively, indicating widespread inequities [3].

Historically, population-level healthcare disparities have been attributed to the racial or ethnic composition of the community residents. However, the conceptual models of health inequity have evolved to include various other dimensions such as sociodemographics, disability, and geographic location [4]. Social Vulnerability Index (SVI), defined by the $\mathrm{CDC}$, is a composite index of 15 variables grouped into four themes providing national and state-specific county ranking [5]. The four underlying themes include "socioeconomic status," "household composition and disability," "minority status and language," and "housing type and transportation" (Fig. 1) [5].

California is the most populous U.S. state with a diverse racial and ethnic composition. Having the nation's highest Diversity Index, California is considered a majority-minority state, as there is no race which constitutes the majority of the population, indicating the highly heterogenous nature of its population. As of 2019, California contains one of the most diverse and highest minority population in the U.S., with only $36.5 \%$ of the population being White, in contrast with $39.4 \%$ being Hispanic or Latinx, 6.5\% being Black or African American, and $15.5 \%$ being Asian. Due to these characteristics, analysis of the vaccination roll-out in California provides a valuable opportunity to investigate the inequities in COVID-19 vaccine distribution among minorities and across heterogenous populations $[6,7]$.

The need for equity in vaccine rollout was recognized in California by dedicating $40 \%$ of doses for the hardest-hit communities (Fig. 2) [8] . Yet, as of as of May 4, 2021, nearly $53 \%$ of individuals in the lowest quartile of the Healthy Places Index remained unvaccinated [9]. Although the gap in healthcare delivery among communities of different social vulnerability status is well-known, to the best of our knowledge, there is no peer-reviewed report on the dynamic trajectories of vaccination in relation to vulnerability indicators in the U.S. Therefore, in this study, we aimed to determine whether the longitudinal trends in vaccination rates across California counties differ by SVI. More specifically, we aimed to model the growth rate in vaccination coverage and the anticipated maximum proportion of vaccinated individuals in relation to county-level overall SVI and its underlying themes.

Under an emergency use authorization issued by the U.S. Food and Drug Administration (FDA), the Pfizer-BioNTech COVID-19 vaccine booster is now allowed for certain populations who are at increased risk of occupational exposure to the virus or developing its complications [10]. Meanwhile, the widespread use of booster shots is being considered by regulatory authorities across the globe. The empirical data obtained during the first round of vaccine rollout represents an opportunity to uncover the pitfalls in distribution and disparities in resource allocation in relation to demands. This real-world data can inform the strategies for equitable delivery of health services in future public health crises, avoid prior mistakes, and ensure the allocation of resources to the most vulnerable populations in the event of unrestricted booster rollout during the current pandemic.

\section{Materials and Methods}

\section{Data Acquisition}

The Social Vulnerability Index (SVI) was created in 2011 by the Agency for Toxic Substances and Disease Registry (ATSDR)'s Geospatial Research, Analysis \& Services

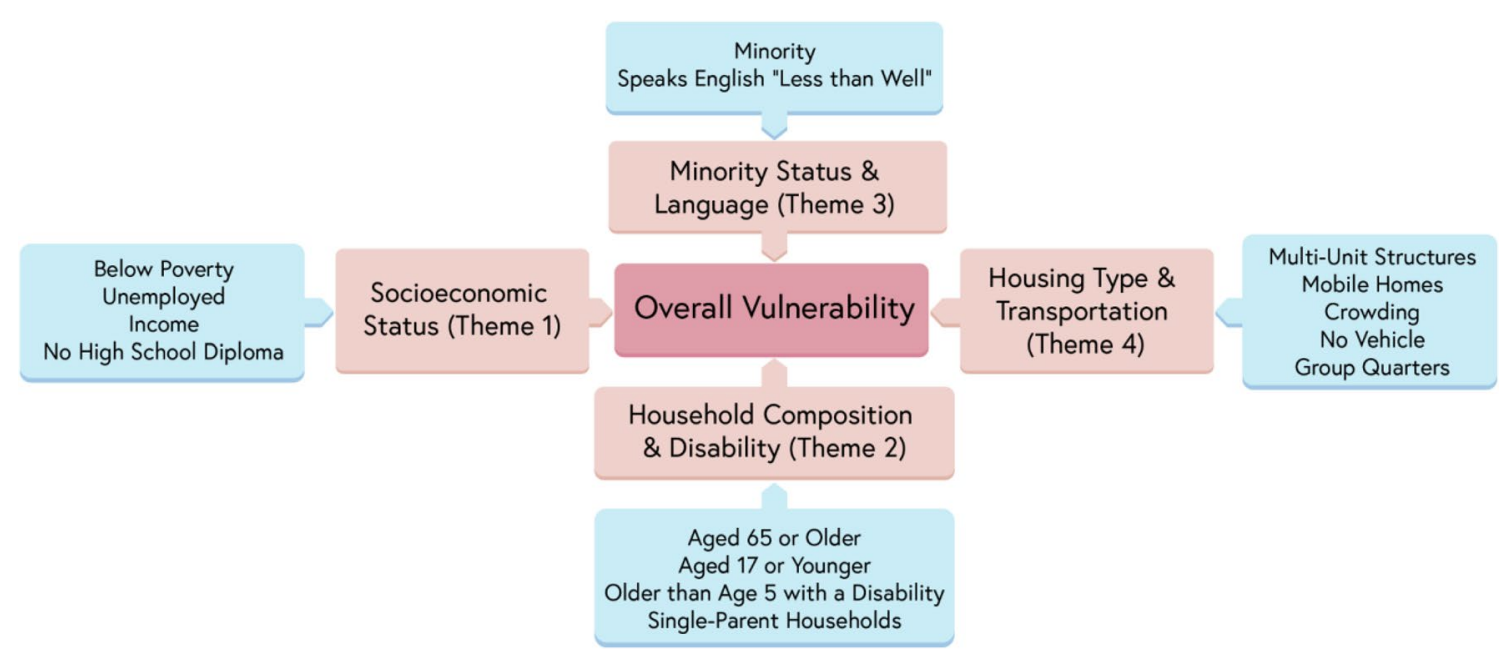

Fig. 1 Social Vulnerability Index (SVI), underlying themes, and components 
Fig. 2 Chronological overview of major national and state-wide (California) vaccination events and policies. Prior to the arrival of the first shipment of Pfizer-BioNTech COVID-19 vaccines in California, the California Department of Public Health (CDPH) issued a statement on December 5, 2020, recommending an initial Phase 1a vaccine allocation for healthcare workers and older or at-risk residents of long-term care facilities. Vaccine supply was limited in the early stages of distribution, and these populations were determined to be at risk of direct exposure to the virus and were prioritized to receive the vaccine first [35]. The recommendation was made anticipating the federal government's vaccine distribution efforts across the nation as the FDA issued Emergency Use Authorizations (EUA) for the PfizerBioNTech vaccine on December 11, 2020 [36] and for the Moderna COVID-19 vaccine on December 18, 2020 [37]. At the time, these vaccines were authorized for individuals aged 16 and older and for individuals aged 18 and older, respectively. With the first shipment of Pfizer-BioNTech vaccines, California Governor Gavin Newsom launched the "Vaccinate all 58" campaign on December 14, 2020 in an effort to distribute vaccines safely, fairly, and equitably for all 58 counties of the state [38]. On January 13, 2021, CDPH issued a statement opening up vaccine eligibility to individuals aged 65 and older. This population was identified as part of Phase $1 \mathrm{~b}$, and while vaccine demand was still much higher than the supply, the demand among healthcare workers declined among the initial Phase 1a populations [39]. Phase $1 \mathrm{~b}$ populations included individuals aged 65 and older as well as workers in food and agriculture, education and childcare services, and emergency services [40]. Because the state gave counties the power to decide when to adopt vaccine phases based on vaccine availability, parts of California moved to Phase $1 \mathrm{~b}$ earlier than others, and the various populations in Phase $1 \mathrm{~b}$ were eligible on different dates across the state. A third vaccine, Johnson \& Johnson/Janssen COVID-19 vaccine, was issued an EUA by the FDA on February 27, 2021 for individuals 18 and older [41]. This further increased vaccine availability in California as vaccine demand continued to outpace supply. A brief pause in the Johnson \& Johnson/ Janssen vaccine starting April 13, 2021 [42] was issued jointly by the FDA and CDC as a result of concerns that the vaccine led to six rare cases of blood clot development, but this pause was lifted on April 23, 2021 jointly by both agencies after a safety review determined that the potential benefits of receiving the Johnson \& Johnson/Janssen vaccine outweighed the potential risks [43]. Further updates to vaccine eligibility for Californians were made in the next two months. On March 11, 2021, vaccine eligibility was extended to residents and workers in facilities such as prisons and homeless shelters (including the homeless population). These populations were determined to be in facilities at great risk of spreading the virus and around individuals likely to have medical conditions that increased the risk of developing negative effects from the virus. Eligibility was also extended to public transport, airport, and commercial airline workers because of their risk of contracting the virus at work. These workers were also determined to be working critical operations [44]. On April 1, 2021, individuals aged 50-64 were eligible to receive vaccines, and Californians aged 16 and older were eligible shortly after beginning April 15, 2021. The Moderna and Johnson \& Johnson vaccines were still authorized for individuals 18 and older only, but the FDA expanded the EUA on the Pfizer-BioNTech vaccine May 10, 2021 for vaccinating individuals aged 12 through 15 [45]. This was followed by $\mathrm{CDPH}$ announcing vaccine eligibility for Californians aged 12 and older beginning May 12, 2021 [40] (LTC: Long-term care, FDA: U.S. Food and Drug Administration, EUA: Emergency Use Authorization, CDC: U.S. Centers for Disease Control and Prevention)
Dec.

5 Phase 1A: Vaccine allocation for healthcare workers and LTC residents

11 FDA approves Pfizer EUA for individuals aged $16+$

14 Governor Newsom launches "Vaccinate all 58" campaign as first shipments of Pfizer vaccine arrive in CA

18 FDA approves Moderna EUA for individuals aged 18+

2021

Jan.

13 Phase 1B: CA prioritizes individuals aged $65+$ as well as workers in food and agriculture, education and childcare

Feb. services, and emergency services

27 FDA approves Johnson \& Johnson EUA

Mar. for individuals aged 18+

11 Vaccine eligibility expanded to additional groups, e.g. transportation, residential facilities, with high risk of occupational exposure*

Apr.

1 Individuals aged 50-64 are eligible

13 CDC and FDA recommend a pause in use of Johnson \& Johnson vaccine

15 Individuals aged 16+ are eligible

23 FDA and CDC lift recommended pause in Johnson \& Johnson vaccine

May

10 FDA expands Pfizer EUA for individuals aged $12+$

12 Individuals aged 12+ are eligible to receive Pfizer

Program (GRASP) and aims to help guide public health officials and emergency response planners in identifying the communities that should be prioritized in the reception of aid $[5,11]$. The SVI provides relative rankings to subregions within the region of interest, for instance, relative rankings of vulnerabilities of the 58 counties in California. This metric provides useful insight for public health officials and local planners for primarily allocating emergency preparedness funding to vulnerable communities, estimating 
resource allocation, and development of supportive strategies for those with special needs, non-English speakers and minorities, older individuals, and those without vehicles or adequate resources to fend off natural catastrophes [5]. Although other similar vulnerability metrics exist, such as the HPI (Healthy Place Index), the SVI is widely reputable and has been adopted by 29 jurisdictions [12-14] across the U.S. to guide COVID-19 responses, and has been utilized in paramount CDC studies [2].

The SVI values used in our study, from the ATSDR, 2018 data [15] were used to measure the levels of vulnerability across California's 58 counties. These values differ from the nationwide data since the ranking is in reference to California counties. Each county is assigned a numerical value between 0 and 1 based on its rank in the state for the overall SVI level and its four themes (Fig. 1) with lower values indicating a lower vulnerability status [5]. Counties were categorized into tertiles for overall SVI and each of its four components: SVI $<0.33$ describing low vulnerability, $0.33 \leq$ SVI $<0.66$ describing moderate vulnerability, and SVI $\geq 0.66$ describing high vulnerability. The vaccination data including the cumulative number of residents who received at least one vaccine dose was collected from the California Health \& Human Services Agency (CHHS) [16].

\section{Data Curation}

R software version 4.0.4 was used to curate and clean the data along with the tidyverse package. To produce the daily proportion of residents vaccinated in each county (p), the cumulative count of residents with at least one dose of any authorized vaccines was used as the numerator. Similar to the CDC's methodology [2], the denominator was defined as the total population of each county from 2019 census estimates [17] For dates with missing data, we used the last available date's value to impute the cumulative daily proportion of residents vaccinated. Then, we merged each county's respective SVI values and tertiles into the dataset. The final dataset contained one data point per county per day for all 58 California counties with a temporal coverage from December 15, 2020 (or the earliest available data) to May 23, 2021.

\section{Modeling Strategy and Statistical Analysis}

Initial analyses were performed to determine the shape of the longitudinal curves of cumulative vaccination status (total with $1+$ dose and total fully vaccinated) for each county. These curves followed that of a logistic growth curve, such as those traditionally used in ecology to describe the growth of a population towards an asymptotic value. This curve describes the value of the population at a particular time as a function of a per capita growth rate ("vaccination velocity") and the carrying capacity ("asymptotic maximum"): [18]
$N_{t}=\frac{K N_{0} e^{v t}}{K+N_{0}\left(e^{v t}-1\right)}$

Using this framework to describe the number of individuals vaccinated, the instantaneous change in the population vaccinated at a given time can be described as:

$\frac{d N}{d t}=v\left(\frac{K-N_{t}}{K}\right) N_{t}$

To fit these equations to our data, we used a nonlinear least-squares approach using the nls function in $\mathrm{R}$ which estimates the parameter values in the specified equation that best fit the data $[19,20]$. Because we were interested in determining whether certain parameters ( $\mathrm{K}$ and $\mathrm{v}$ ) varied based on SVI, we included dummy variable interactions in the model to allow for these parameter estimates to differ among SVI groups. To standardize the outcome across counties with different population sizes, we used the proportion of individuals vaccinated (p) as the outcome. Due to the very low initial proportion of individuals vaccinated, we treated the starting proportion $\mathrm{p}_{0}$ as a nuisance parameter and did not test for differential effects in $\mathrm{p}_{0}$ by SVI status. We constructed SVI indicator variables $i_{1}$ and $i_{2}$ being indicators of a county having "moderate" and "high" SVI status, respectively. With $\mathrm{dk}_{1}$ and $\mathrm{dk}_{2}$ capturing the difference in the $\mathrm{k}$ term for counties with Moderate and High SVI status, respectively, and $d v_{1}$ and $\mathrm{dv}_{2}$ capturing the respective differences in the $\mathrm{v}$ term, the final equation we fit was:

$$
p_{t}=\frac{\left(K+d K_{1} * i_{1}+d K_{2} * i_{2}\right) p_{0} e^{\left(v+d v_{1} * i_{1}+v d_{2} * i_{2}\right) t}}{\left(K+d K_{1} * i_{1}+d K_{2} * i_{2}\right)+p_{0}\left(e^{\left(v+d v_{1} * i_{1}+d v_{2} * i_{2}\right) t}-1\right)}
$$

Estimated $\mathrm{v}$ and $\mathrm{K}$ parameters for low, moderate, and high SVI counties were computed using the lincom function (biostat3 package).

To determine whether vaccination disparities by SVI status in early 2021 persisted through time, we used negative binomial regression models to examine the rate ratios of proportion vaccinated in low- and moderate- versus high-SVI counties at three separate "snapshots" in time: January 1, March 1, and May 1, 2021. In these models we used cumulative number of individuals vaccinated as the outcome, with an offset of the natural log of county population size, thus effectively modeling the rate of individuals vaccinated at each county. Models included an interaction term between SVI category and date, to test whether the proportional differences in vaccination rates persisted throughout the study period. To provide additional context, we reported the instantaneous coverage velocity at each date, calculated as $\mathrm{dP} / \mathrm{dt}=\mathrm{r}\left(\left(\mathrm{K}-\mathrm{P} \_\mathrm{t}\right) / \mathrm{K}\right) \mathrm{P} \_\mathrm{t}$ where $\mathrm{P}_{-} \mathrm{t}$ is the proportion vaccinated at time $\mathrm{t}$. 


\section{Results}

\section{Data Summary}

A total of 9228 data points from 58 California counties were analyzed, with 258 missing data points $(2.79 \%)$, many of which fell on holidays and weekends when a lag in reporting likely occurred. Distribution of vaccination coverage at the study endpoint and overall SVI ranks are depicted in Fig. 3. The average county population estimates were comparable among the three categories of overall SVI ( $P$-value range: 0.76-0.1, Supplementary Fig. 1). At the study endpoint, the average proportion of vaccinated population in counties with low, moderate, and high overall SVI was $0.54 \pm 0.10,0.48 \pm 0.13$, and $0.40 \pm 0.08$, respectively, with a lower proportion in high vulnerability category as compared to moderate $(P=0.0351)$ and low $(P=0.0003)$ categories (Supplementary Fig. 1).

\section{Model Performance}

The nonlinear ecological growth curve approach allowed a meaningful hypothesis testing of the $\mathrm{v}$ and $\mathrm{K}$ parameters across counties of differing SVI status. While allowing for more flexible modeling, the use of cubic splines to describe longitudinal curves does not allow the testing of differences in growth and asymptotic maximum parameters based on SVI. Therefore, to assess the acceptability of our models, we compared their fit to that of alternate cubic spline models. The $\mathrm{R}^{2}$ values of growth
Fig. 3 Distribution of overall Social Vulnerability Index ranks across 58 California counties and the cumulative rate of individuals who received at least one dose of vaccine per 100,000 capita (circles) at the study endpoint. Numbers in the circles represent the vaccination rate

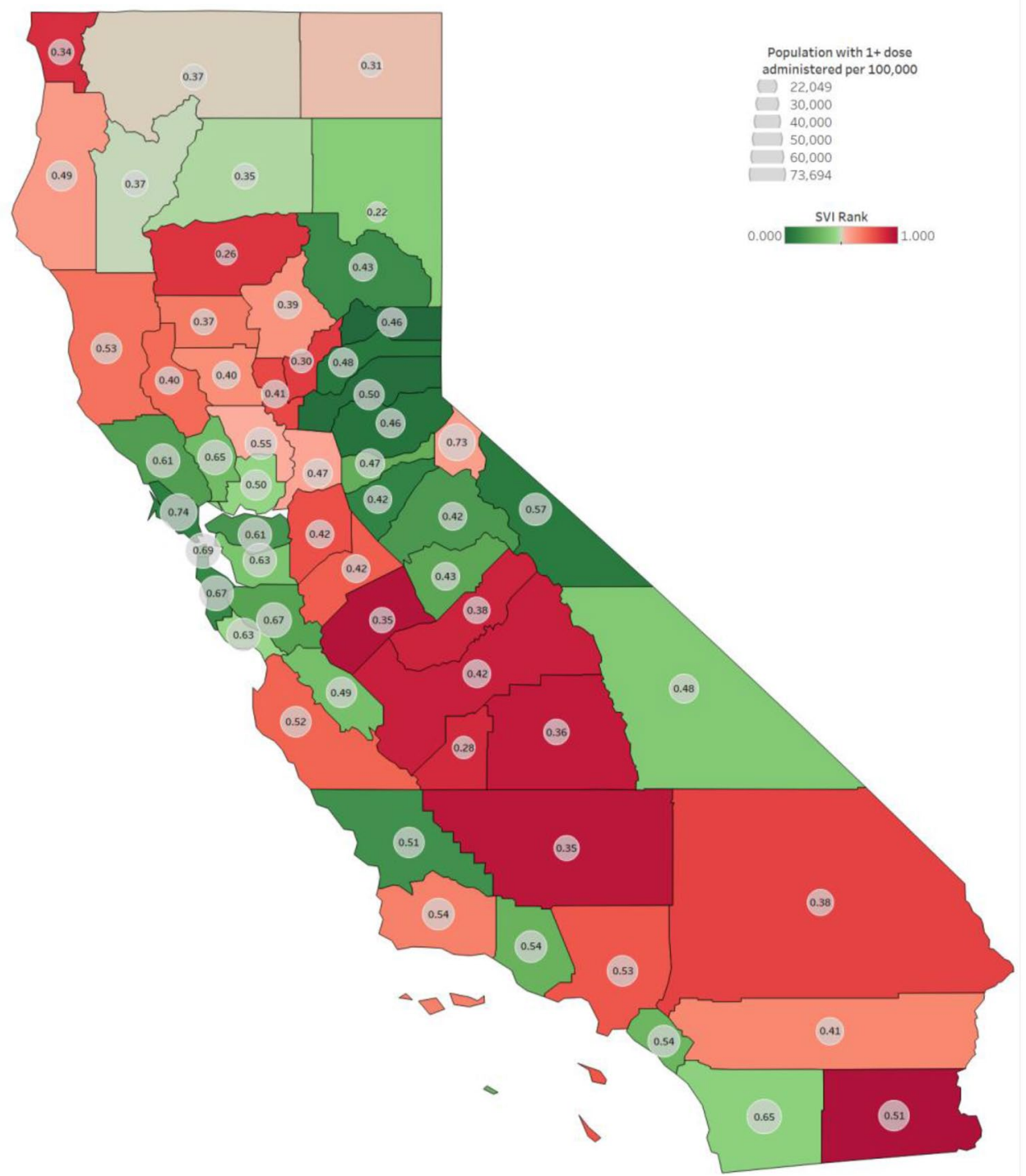


curve models were compared to the "gold standard" cubic spline models and the "null" ecological growth curve model without an effect of SVI. The $\mathrm{R}^{2}$ values of the fit ecological growth models were nearly identical to the cubic spline models, suggesting these models fit the data as well as the more complex alternative. Additionally, the $\mathrm{R}^{2}$ values for the models with the effect of SVI $\left(R^{2}: 0.816-0.877\right)$ were higher than the null model $\left(\mathrm{R}^{2}=0.813\right)$, suggesting meaningful differences in longitudinal curves among counties of differing SVI status. Overall, the inclusion of SVI Theme 2 led to the best explanation of differences in proportion vaccinated across time $\left(R^{2}=0.877\right)$, while SVI Theme 4 was the poorest at explaining why proportion vaccinated differed among counties across time $\left(\mathrm{R}^{2}=0.816\right)$. Residuals for each of the models appeared normally distributed with no outliers. Two counties consistently had standardized residuals greater than 3 in magnitude: Alpine County with consistently high proportion vaccinated versus predicted, and Lassen County with consistently low proportion vaccinated versus predicted. These counties did not appear to have a large effect on the estimate of the mean vaccination proportion. The final models are depicted in Fig. 4.

\section{Vaccination Coverage Velocity (v)}

The $\mathrm{v}$ parameter estimates were significantly different among the overall SVI categories, indicating an overall gap in coverage across the SVI categories over time, with similar trends observed for Themes 1, 2, and 4 (Fig. 4, Table 1). However, the velocity of vaccination coverage was comparable between low and moderate categories when counties were categorized according to Theme $3(v=0.042$ and 0.041 , respectively). Counties within the low category of this theme, representing those with a larger proportion of non-minority and English-speaking individuals, demonstrated the highest coverage velocity across all themecategory combinations. Meanwhile, grouping counties by Theme 3 demonstrated the largest disparity in coverage velocity between the low and high vulnerability categories ( v $=0.042$ vs. 0.035 respectively, $P<0.001$ ).

\section{Vaccination Asymptotic Maximum (K)}

Based on the current trajectory, the asymptotic maximum for counties in the low overall SVI category is estimated to be 0.561 (95\% CI 0.551, 0.571; Fig. 5, Table 1). This parameter was lower in counties with moderate $(\mathrm{K}=0.504$, $95 \%$ CI $0.494,0.515)$ and high $(\mathrm{K}=0.462,95 \%$ CI 0.448 ,

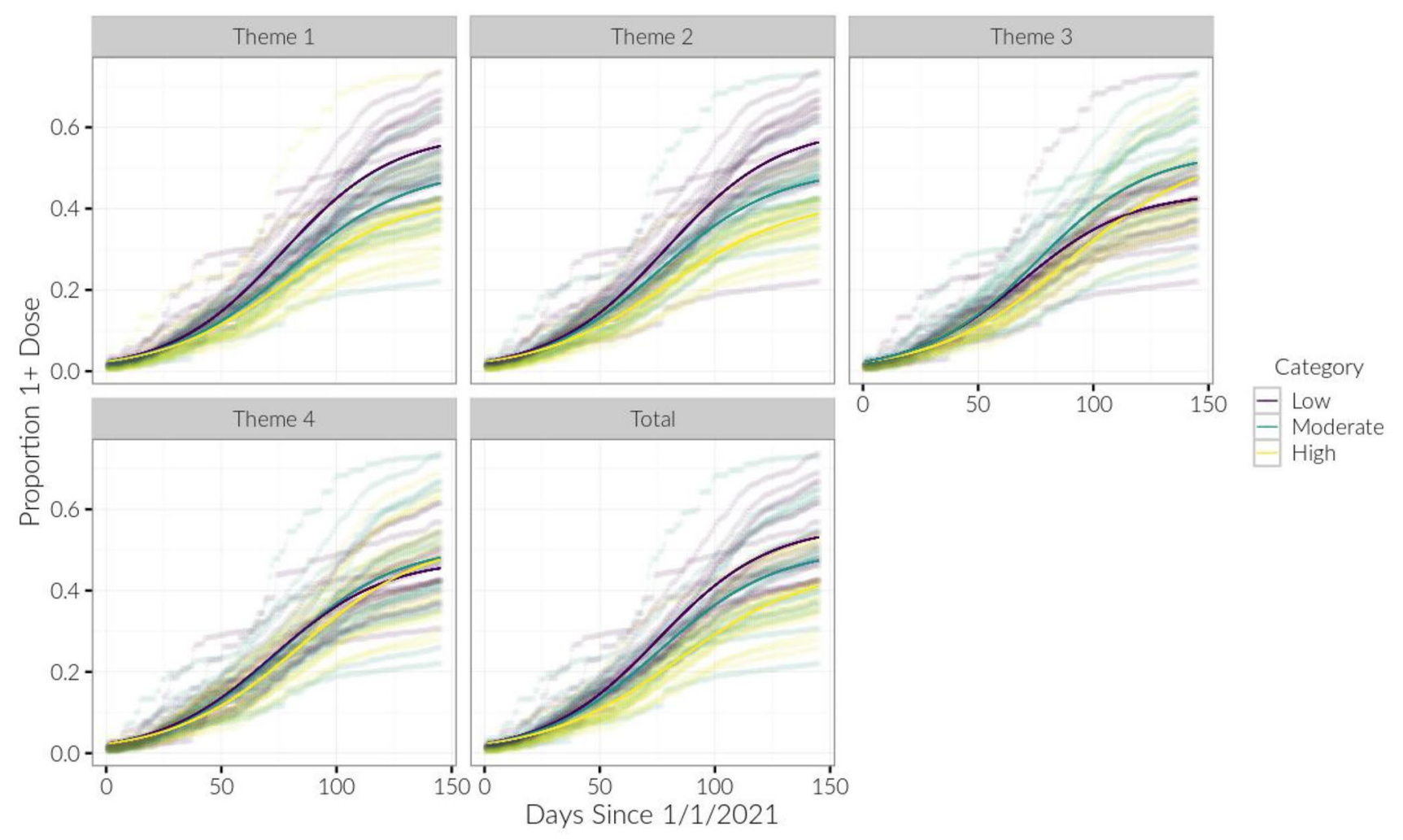

Fig. 4 Proportion of persons with 1+vaccination dose is shown for each county, with the model-fit estimates for each Social Vulnerability Index (SVI) category shown in bold 
Table 1 Parameter estimates for each SVI category, for each theme, with 95\% confidence intervals

\begin{tabular}{|c|c|c|c|c|c|}
\hline $\begin{array}{l}\text { Parameter } \\
\text { / SVI Cat- } \\
\text { egory }\end{array}$ & Overall & Theme1 & Theme2 & Theme3 & Theme4 \\
\hline \multicolumn{6}{|l|}{$N_{0}$} \\
\hline & $0.0238(0.0223,0.0252)$ & $0.0239(0.0225,0.0254)$ & $0.024(0.0227,0.0254)$ & $0.0229(0.0213,0.0244)$ & $0.0231(0.0215,0.0247)$ \\
\hline \multicolumn{6}{|l|}{$v$} \\
\hline Low & $0.0414(0.0403,0.0425)$ & $0.0413(0.0402,0.0423)$ & $0.0404(0.0394,0.0414)$ & $0.0423(0.0409,0.0436)$ & $0.0412(0.0398,0.0426)$ \\
\hline Mod & $0.0396(0.0385,0.0408)$ & $0.0376(0.0365,0.0387)$ & $0.0392(0.0381,0.0402)$ & $0.0413(0.0401,0.0426)$ & $0.0399(0.0386,0.0413)$ \\
\hline High & $0.0346(0.0334,0.0358)$ & $0.0364(0.0352,0.0376)$ & $0.0355(0.0343,0.0366)$ & $0.035(0.0338,0.0362)$ & $0.0365(0.0353,0.0378)$ \\
\hline \multicolumn{6}{|l|}{$K$} \\
\hline Low & $0.561(0.551,0.571)$ & $0.587(0.577,0.597)$ & $0.602(0.592,0.612)$ & $0.441(0.432,0.45)$ & $0.478(0.468,0.488)$ \\
\hline Mod & $0.504(0.494,0.515)$ & $0.502(0.491,0.514)$ & $0.501(0.491,0.51)$ & $0.542(0.531,0.552)$ & $0.512(0.5,0.523)$ \\
\hline High & $0.462(0.448,0.476)$ & $0.436(0.425,0.447)$ & $0.425(0.413,0.436)$ & $0.544(0.527,0.561)$ & $0.529(0.514,0.544)$ \\
\hline$R^{2}$ & 0.857 & 0.867 & 0.877 & 0.833 & 0.816 \\
\hline $\mathrm{R}^{2} \mathrm{Null}$ & 0.813 & 0.813 & 0.813 & 0.813 & 0.813 \\
\hline $\mathrm{R}^{2}$ Spline & 0.858 & 0.867 & 0.878 & 0.834 & 0.817 \\
\hline
\end{tabular}

The $\mathrm{N}_{0}$ parameter was modeled as static across the SVI categories. $\mathrm{R}^{2}$ values are listed for the current models, the null model (i.e., logistic growth without an effect of SVI), and a cubic spline model. Within each model, the parameter estimate was always significantly different $(\mathrm{P}<.05)$ among SVI categories except for the following: 1) in Theme 3, $v$ was not different between Low and Mod categories, 2) in Theme 3, $K$ was not different between Mod and High categories

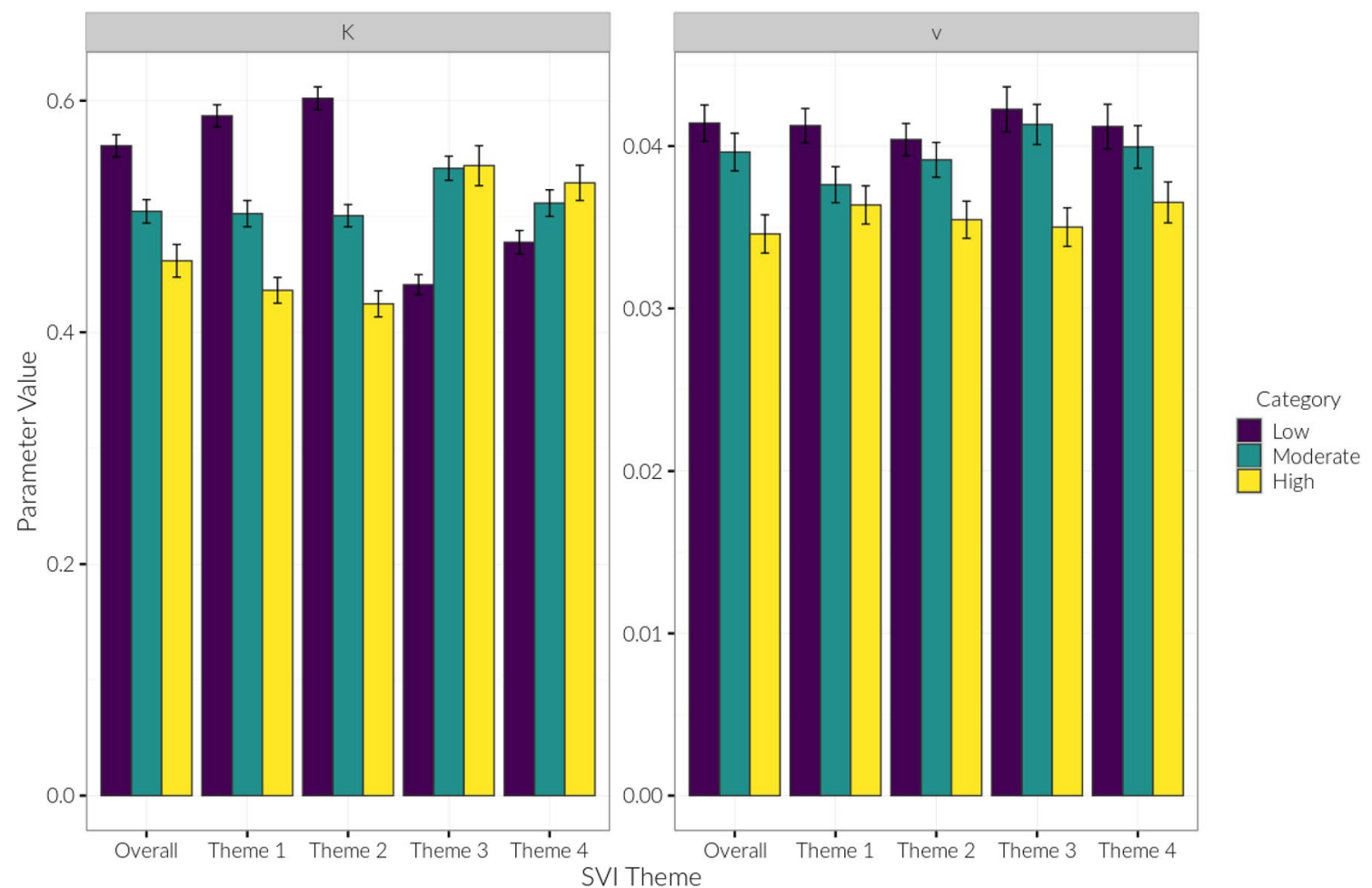

Fig. 5 Model estimates of K and v parameters for each theme and Social Vulnerability Index (SVI) category, with 95\% confidence intervals

0.476) overall SVI. The models demonstrated similar patterns for themes 1 and 2 . However, based on the models constructed using themes 3 and 4, there was an inverse relationship between the vulnerability categories and K estimates.
Among models with differing SVI themes, the K estimate was always different (all $P \mathrm{~s}<0.001$ ) among vulnerability categories, except for Theme 3 which yielded comparable values between moderate and high categories $(P=0.06)$. 
Among all theme-category combinations, counties in low vulnerability category of Theme 2 were estimated to achieve the largest asymptotic maximum $(\mathrm{K}=0.602,95 \%$ CI 0.592 , 0.612 ), while themes 1 and 2 yielded the largest disparity: when counties were categorized according to Theme 1, the $\mathrm{K}$ parameters for low and high categories were 0.587 (95\% CI $0.577,0.597)$ and 0.436 (95\% CI $0.425,0.447)$, respectively $(P<0.001)$.

\section{"Snapshots" Analysis}

Overall, we observed large initial disparities in vaccination rates by SVI status on January 1, which appeared to become attenuated over time. For overall SVI status, on January 1, low-vulnerability counties had 1.75 (95\% CI 1.44, 2.12) times the cumulative vaccination rates as high vulnerability counties (Fig. 6, Table 2). This disparity significantly decreased to $1.35(95 \%$ CI $1.11,1.63)$ times the cumulative vaccination rates by May $1(P=0.03)$.

There was a large initial disparity in cumulative vaccination rates by SVI Theme 1. On January 1, low-vulnerability counties had $1.91(95 \%$ CI $1.58,2.31)$ times the cumulative vaccination rates as high-vulnerability counties, which decreased to $1.42(95 \%$ CI $1.18,1.71)$ times by May 1 $(\mathrm{P}=0.01)$. Regarding Theme 3 , low-vulnerability counties began the year with $1.42(95 \%$ CI $1.15,1.75)$ times the cumulative vaccination rates as high-vulnerability counties, but by May 1 this rate ratio decreased $(P=0.002)$ effectively yielding no difference in cumulative vaccination $(R R=0.95$, 95\% CI 0.77, 1.17). Trends in decreasing rate ratios over time, suggesting more equal cumulative vaccination rates by SVI status over time, were seen for the other themes. The instantaneous coverage velocity corresponding to each date is outlined in Table 3 .

\section{Discussion}

The COVID-19 pandemic shed a broader light on the existing gaps in healthcare delivery and outcomes across the socio-demographic subgroups in the U.S. This study was performed to determine the vaccination coverage velocity of California counties based on the underlying social vulnerability status, and to utilize the longitudinal trends to estimate the disparities in maximum anticipated proportion of vaccinated individuals based on the current

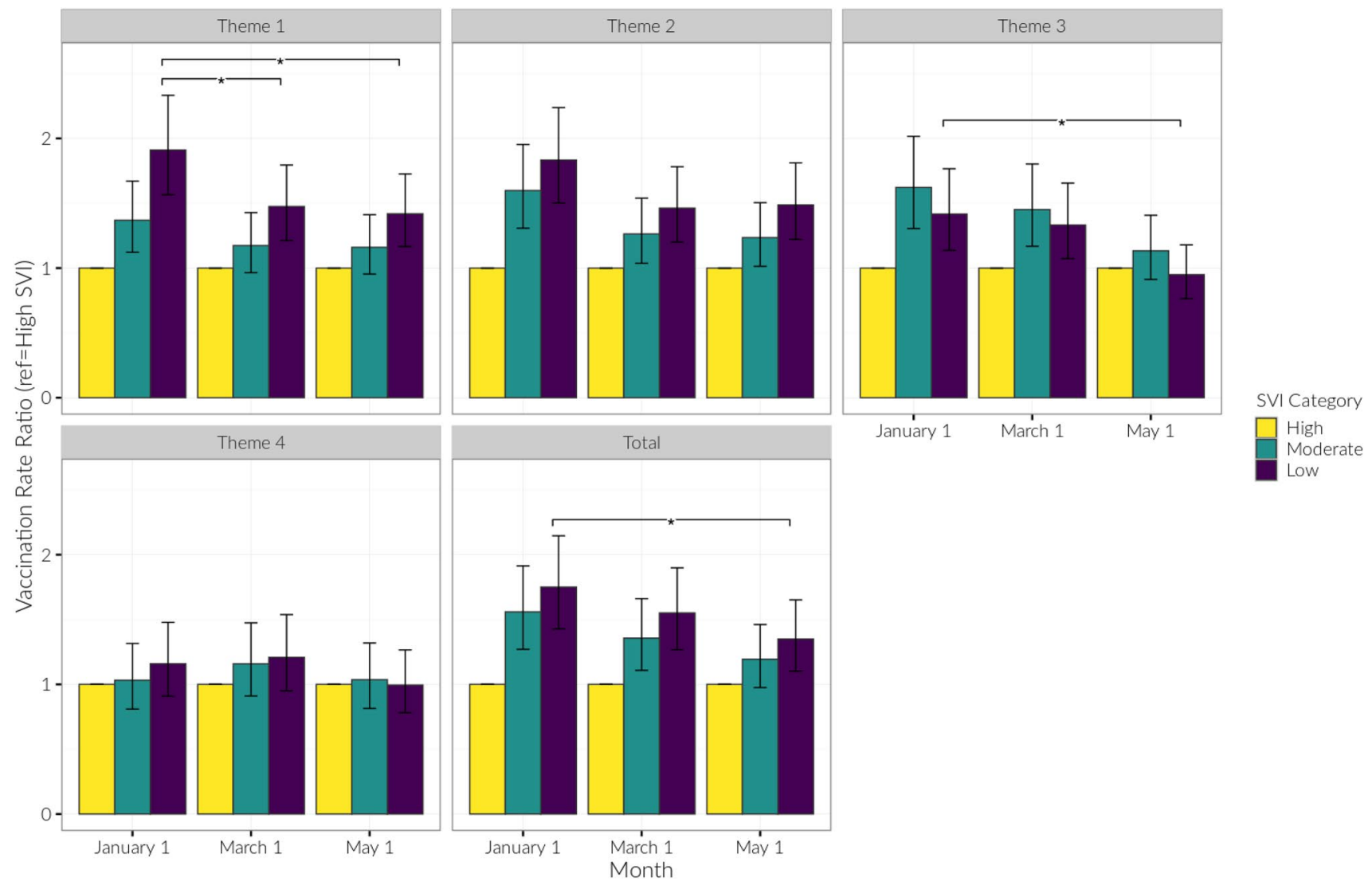

Fig. 6 Vaccination rate ratios for low and moderate SVI vs. high SVI counties, by date and SVI category. Presented are rate ratios with 95\% confidence intervals. *Indicates a temporal reduction in the rate ratio for the given date vs. January 1 at $\mathrm{p}<0.05$ 
Table 2 Snapshot analysis of trends in rate ratio of proportion vaccinated in low and moderate versus high SVI counties using negative binomial regression models

\begin{tabular}{lll}
\hline SVI theme/Date & \multicolumn{2}{l}{ Vaccination rate ratio $(95 \% \mathrm{CI})$} \\
\cline { 2 - 3 } & Low vs. high & Moderate vs. high \\
\hline Overall SVI & & \\
January 1 & $1.75(1.44,2.12)$ & $1.56(1.28,1.89)$ \\
March 1 & $1.55(1.28,1.88)$ & $1.36(1.12,1.64)$ \\
May 1 & $1.35(1.11,1.63)^{*}$ & $1.19(0.99,1.45)$ \\
Theme 1 & & \\
January 1 & $1.91(1.58,2.31)$ & $1.37(1.13,1.65)$ \\
March 1 & $1.47(1.22,1.78)^{*}$ & $1.17(0.98,1.41)$ \\
May 1 & $1.42(1.18,1.71)^{*}$ & $1.16(0.96,1.40)$ \\
Theme 2 & & \\
January 1 & $1.83(1.52,2.21)$ & $1.60(1.32,1.93)$ \\
March 1 & $1.46(1.21,1.76)$ & $1.26(1.05,1.52)$ \\
May 1 & $1.49(1.23,1.79)$ & $1.23(1.02,1.49)$ \\
Theme 3 & & \\
January 1 & $1.42(1.15,1.75)$ & $1.62(1.32,1.99)$ \\
March 1 & $1.33(1.08,1.64)$ & $1.45(1.18,1.78)$ \\
May 1 & $0.95(0.77,1.17)^{*}$ & $1.13(0.92,1.39)$ \\
Theme 4 & & \\
January 1 & $1.16(0.92,1.46)$ & $1.03(0.82,1.30)$ \\
March 1 & $1.21(0.96,1.52)$ & $1.16(0.92,1.46)$ \\
May 1 & $0.99(0.79,1.25)$ & $1.04(0.83,1.30)$ \\
\hline andicas &
\end{tabular}

* Indicates a temporal reduction in the rate ratio for the given date versus January 1st, 2021, at $\mathrm{P}<0.05$. SVI, Social Vulnerability Index

trajectory. Using the data from 58 California counties, we demonstrated a decreasing yet significant gap in vaccination coverage based on the overall SVI status. Counties with high vulnerability designation according to minority status and language (Theme 3 ) demonstrated the lowest coverage velocity across all theme-category combinations. Meanwhile, counties in this category have suffered from the highest average mortality rate as of May 23, 2021, when compared to moderate and low categories.

The large initial disparities in vaccination rates by overall SVI status, Theme 1, and Theme 3 attenuated over time, while counties in the high vulnerability category according to Themes 3 and 4 are estimated to eventually achieve a higher proportion of residents vaccinated compared to less vulnerable counterparts. Given the higher proportion of Black and Hispanic populations in the high vulnerability category of Theme 3 , our findings parallel those of a nation-wide study which showed that counties with large Hispanic and Black populations have a higher vaccination uptake [21]. It seems that when these populations faced challenges to accessibility, a low vaccine coverage velocity was observed, while improved accessibility led to a greater uptake.
Table 3 Predicted instantaneous coverage velocity, by date and SVI category

\begin{tabular}{|c|c|c|c|}
\hline \multirow[t]{2}{*}{ SVI theme/Date } & \multicolumn{3}{|c|}{ Predicted instantaneous velocity $(\mathrm{dP} / \mathrm{dt})^{\mathrm{a}}$} \\
\hline & Low & Moderate & High \\
\hline \multicolumn{4}{|l|}{ Overall SVI } \\
\hline January 1 & 0.098 & 0.093 & 0.093 \\
\hline March 1 & 0.523 & 0.457 & 0.394 \\
\hline May 1 & 0.264 & 0.245 & 0.273 \\
\hline \multicolumn{4}{|l|}{ Theme 1} \\
\hline January 1 & 0.098 & 0.089 & 0.088 \\
\hline March 1 & 0.555 & 0.451 & 0.376 \\
\hline May 1 & 0.287 & 0.272 & 0.228 \\
\hline \multicolumn{4}{|l|}{ Theme 2} \\
\hline January 1 & 0.097 & 0.093 & 0.092 \\
\hline March 1 & 0.572 & 0.452 & 0.361 \\
\hline May 1 & 0.316 & 0.247 & 0.228 \\
\hline \multicolumn{4}{|l|}{ Theme 3} \\
\hline January 1 & 0.095 & 0.094 & 0.094 \\
\hline March 1 & 0.376 & 0.506 & 0.476 \\
\hline May 1 & 0.166 & 0.258 & 0.355 \\
\hline \multicolumn{4}{|l|}{ Theme 4} \\
\hline January 1 & 0.094 & 0.091 & 0.091 \\
\hline March 1 & 0.426 & 0.470 & 0.476 \\
\hline May 1 & 0.206 & 0.253 & 0.319 \\
\hline
\end{tabular}

${ }^{\mathrm{a}}$ For interpretation, metric is multiplied by 100 and is interpreted as the change in percent vaccinated per day at the given date. Instantaneous coverage velocity is calculated as: $d P / d t=r\left(\left(K-P_{t}\right) / K\right) P_{t}$ where $P_{t}$ is the proportion vaccinated at time $\mathrm{t}$

The higher estimated asymptotic maximums in vulnerable counties can be attributed to California-funded target outreach programs and increased vaccine allocation to the most vulnerable populations often consisting of higher minority populations. The increases in proportion of residents vaccinated in low and moderately vulnerable counties according to Theme 3 began to show a plateau near the 100th day of 2021, while the pace of coverage was relatively maintained in highly-vulnerable counties (Fig. 4). Additionally, the vaccination rate ratios for low and moderate SVI Theme 3 counties diminished from March to May, reaching close to 1.0 in reference to high-vulnerability counties. This change in trend occurred nearly concomitantly with California's fervent spending in community outreach programs, media, and increased vaccine allocation for the hardest-hit communities in March 2021 [22]. Similar findings have been reported in Maryland, suggesting that targeting communities based on vulnerability metrics can help mitigate health inequities [23].

Racial/ethnic minority populations are more likely to live in multigenerational households or in more crowded neighborhoods and units [24, 25]. Latinx persons in California are 8.1 times more likely to live in households facing 
exposure risks like living in households with essential workers and having fewer rooms than household members [26]. These overlapping characteristics shared between Themes 3 and 4 may explain their similarity in trends of asymptotic maximum.

Vaccine disparities across all SVI themes have decreased over time (Fig. 6) likely due to accessibility becoming more ubiquitous with programs targeting vulnerable populations. To mitigate the vaccine inequities, elimination of barriers to vaccination is just as important, if not more, than setting equitable vaccine eligibility and prioritization criteria. Individuals of an ethnic/racial minority are typically over-represented among those with essential public-facing occupations which were prioritized during the initial phases of vaccination [25]. Yet, some workers were likely unable to receive the vaccine due to a multitude of barriers, such as concerns about time off of work. Furthermore, uninsured individuals may have had concerns about the healthcare costs associated with vaccine side effects, while immigrants may have faced challenges due to the vaccination data being tracked by personal identifiers, fearing their immigration status would be jeopardized [27].

Although many studies suggest inaccessibility as a significant factor behind vaccination inequities, the negative contribution of vaccine hesitancy cannot be fully discounted [23]. A longitudinal survey of vaccine acceptance showed that in November 2020, only $42 \%$ of African American respondents expressed their willingness to receive the vaccine, compared to $61 \%$ of White respondents [28]. Another study conducted between March 24, 2020, and February 16, 2021, yielded similar results; compared with White participants, the odds ratios for vaccine hesitancy were 3.15 for Black participants and 1.42 for Hispanics [29]. Although earlier vaccine hesitancy surveys can potentially explain the initial higher vaccine disparities among the Theme 3 categories, more recent surveys show similar levels of willingness to get vaccinated across Black, Hispanic, and White populations [30-32]. This could be a contributing factor to the amelioration of vaccine inequity witnessed in high-vulnerability Theme 3 counties from March to May 2021 (Fig. 4, Fig. 6) and can potentially be a result of outreach programs like lotteries and mobile vaccine clinics [33]. While a recent study has shown that misinformation can lead to significant declines in willingness to get vaccinated [34], California's outreach and media spending consist of public education programs in efforts to combat such misinformation and better educate more vulnerable residents, which can also lead to increases of willingness to get vaccinated [22].

As part of California's public education campaign, the state planned to support a statewide community outreach program where "trusted messengers" such as communitybased organizations and prominent leaders actively engage with the hardest hit communities, providing individuals with accurate and trusted information resources pertaining to COVID-19 [22]. Moreover, media campaigns were planned to "create culturally competent in-language content," while news briefings and daily news stories were held aiming to engage top public health officers and community leaders to tackle misinformation while reinforcing the public trust in vaccine safety and efficacy [22].

Our study showed that the overall SVI and its four themes are useful in distinguishing differing vaccination rates across counties. As SVI identifies counties with the most vulnerable populations, it can be used for mitigating health inequities and has been adopted by 29 jurisdictions across the U.S. to guide the COVID-19 vaccine distribution [12-14]. Our model for Theme 2 (household composition and disability) demonstrated the best performance, while Theme 3 (minority status and language) yielded the highest discriminative value in terms of vaccination coverage velocity, suggesting this theme as the most informative underlying domain of SVI in the context of COVID-19 vaccination. Yet, more empirical data are needed to unfold the contribution of each SVI category to health outcomes.

\section{Limitations}

The staged vaccine rollout may have affected the study findings, particularly those related to SVI Theme 2 , since counties with a higher proportion of individuals 65 may have achieved a higher early vaccination coverage. Furthermore, members of certain occupations with increased risk of exposure to the virus were initially prioritized. Due to limitations in data availability regarding the demographics of such individuals, we were unable to account for these subgroups in our analysis. Moreover, the SVI does not include some of the underlying factors that contributed to disproportionate vaccination coverage, such as internet access and digital literacy. In terms of dataset limitations, daily vaccination numbers were missing in some data points and were handled by imputing the latest available day's data. Given the small number of missing data points $(258 / 9228,2.79 \%)$ and the nature of our analysis, this limitation is unlikely to have introduced significant bias. Future analyses should confirm whether vaccination trajectories will approach the estimated asymptotic maximums predicted in this study.

\section{Conclusion}

This study provides insight into the problem of COVID19 vaccine disparity across California which can be used to help promote equity during the current pandemic and guide the allocation of future vaccines such as COVID-19 booster shots. By addressing the relationship between SVI 
components with longitudinal trends of vaccine administration, we demonstrated their utility in development of targetbased equitable strategies.

Supplementary Information The online version contains supplementary material available at https://doi.org/10.1007/s10903-021-01308-2.

Funding This study was supported by the National Science Foundation (NSF) under Award Number 2027456. This publication was supported by grants UL1TR001855 and UL1TR000130 from the National Center for Advancing Translational Science (NCATS) of the U.S. National Institutes of Health (NIH). The content is solely the responsibility of the authors and does not necessarily represent the official views of the NSF, NIH, or other entities.

\section{Declarations}

Conflict of interest The authors declare no conflict of interest. No financial disclosures were reported by the authors of this paper.

Ethics Approval Ethical approval was not acquired nor applicable for this research article. No human or animal participants were involved in the study. Publicly available data was used in the process of this study.

\section{References}

1. Risk for COVID-19 Infection, Hospitalization, and Death by Race/ Ethnicity. Centers for Disease Control and Prevention (CDC). Published June 17, 2021. https://www.cdc.gov/coronavirus/2019ncov/covid-data/investigations-discovery/hospitalization-deathby-race-ethnicity.html. Accessed July 14, 2021

2. Hughes MM, Wang A, Grossman MK, et al. County-level COVID19 vaccination coverage and social vulnerability - United States, December 14, 2020-March 1, 2021. MMWR Morb Mortal Wkly Rep. 2021;70(12):431-6. https://doi.org/10.15585/mmwr.mm701 $2 \mathrm{e} 1$.

3. Ndugga N, Pham O, Artiga S, Parker N. Latest Data on COVID19 Vaccinations by Race/Ethnicity. Kaiser Family Foundation (KFF). Published 2021. https://www.kff.org/coronavirus-covid19/issue-brief/latest-data-on-covid-19-vaccinations-race-ethni city/. Accessed July 14, 2021

4. National Academies of Sciences, Engineering, and Medicine, Health and Medicine Division, Board on Population Health and Public Health Practice, Committee on Community-Based Solutions to Promote Health Equity in the United States. Communities in Action: Pathways to Health Equity. (Baciu A, Negussie Y, Geller A, Weinstein JN, eds.). National Academies Press (US); 2017. http://www.ncbi.nlm.nih.gov/books/NBK425848/. Accessed July 14,2021

5. Centers for Disease Control and Prevention (CDC). CDC SVI 2018 Documentation. Published online January 31, 2020. https:// www.atsdr.cdc.gov/placeandhealth/svi/documentation/pdf/SVI20 18Documentation-H.pdf. Accessed June 14, 2021

6. Population Clock. https://www.census.gov/popclock/. Accessed October 11, 2021

7. U.S. Census Bureau QuickFacts: California; United States. https:// www.census.gov/quickfacts/fact/table/CA,US/PST045219. Accessed October 11, 2021

8. California Leads with Public Health and Vaccine Equity to Safely and Sustainably Reopen. Office of Governor Gavin Newsom. Published March 4, 2021. https://www.gov.ca.gov/2021/03/04/calif ornia-leads-with-public-health-and-vaccine-equity-to-safely-andsustainably-reopen/. Accessed July 14, 2021

9. As California Surpasses 30 Million Vaccines, Governor Newsom Doubles Down on Efforts to Vaccinate Hard-to-Reach Communities. Office of Governor Gavin Newsom. Published May 4, 2021. https://www.gov.ca.gov/2021/05/04/as-california-surpasses-30million-vaccines-governor-newsom-doubles-down-on-efforts-tovaccinate-hard-to-reach-communities/. Accessed July 1, 2021

10. Commissioner $\mathrm{O}$ of the. FDA Authorizes Booster Dose of PfizerBioNTech COVID-19 Vaccine for Certain Populations. U.S. Food and Drug Administration (FDA). Published September 22, 2021. https://www.fda.gov/news-events/press-announcements/fdaauthorizes-booster-dose-pfizer-biontech-covid-19-vaccine-certa in-populations. Accessed October 11, 2021

11. At A Glance: CDC/ATSDR Social Vulnerability Index I Place and Health I ATSDR. Published August 30, 2021. https://www. atsdr.cdc.gov/placeandhealth/svi/at-a-glance_svi.html. Accessed October 11, 2021

12. National Academies of Sciences, Engineering, and Medicine. In: Framework for equitable allocation of COVID-19 vccine. The National Academies Press, 2020, pp. 89-144. https://doi.org/10. $17226 / 25917$

13. Schmidt $\mathrm{H}$, Weintraub R, Williams MA, et al. Equitable allocation of COVID-19 vaccines in the United States. Nat Med. 2021;27(7):1298-307. https://doi.org/10.1038/ s41591-021-01379-6.

14. COVID-19 Vaccine Equity in Minnesota. Minessota Department of Public Health. https://www.health.state.mn.us/diseases/coron avirus/vaccine/mnsvi.html. Accessed July 14, 2021

15. CDC/ATSDR SVI Data and Documentation Download I Place and Health I ATSDR. Agency for Toxic Substances and Disease Registry (ASTDR). Published April 28, 2021. https://www.atsdr. cdc.gov/placeandhealth/svi/data_documentation_download.html. Accessed July 14, 2021

16. Statewide COVID-19 Vaccines Administered By County. California Health and Human Services Open Data. https://data.chhs. ca.gov/dataset/vaccine-progress-dashboard/resource/130d7ba2b6eb-438d-a412-741bde207e1c. Accessed May 24, 2021

17. American Community Survey 5-Year Data (2009-2019). The United States Census Bureau. https://www.census.gov/data/devel opers/data-sets/acs-5year.html. Accessed July 14, 2021

18. Rye C, Wise R, Jurukovski V, DeSaix J, Choi J, Avissar Y. Biology. OpenStax; 2016. https://openstax.org/books/biology/pages/1introduction

19. Bates DM, Chambers JM. Nonlinear models. In: Chambers JM, Hastie TJ, editors. Statistical models. Belmont: Wadsworth \& Brooks/Cole; 1992.

20. Bates DM, Watts DG. Nonlinear regression analysis and its applications. New York: Wiley; 1988.

21. Chernyavskiy P, Richardson JW, Ratcliffe SJ. COVID-19 vaccine uptake in United States counties: geospatial vaccination patterns and trajectories towards herd immunity. medRxiv. 2021. https:// doi.org/10.1101/2021.05.28.21257946.

22. FACT SHEET: Ending the Pandemic Through Equitable Vaccine Administration. Published online 2021. https://www.gov.ca.gov/ wp-content/uploads/2021/03/Equitable-Vaccine-AdministrationFact-Sheet.pdf. Accessed July 1, 2021

23. Andrew M, Kavitha R, Maulik J. Vaccine Equity Index Shows Reduction in Maryland Covid-19 Vaccination Disparity in Less Than Two Months. NEJM Catalyst Innovations in Care Delivery. Published online April 23, 202. https://catalyst.nejm.org/ doi/full/https://doi.org/10.1056/CAT.21.01261. Accessed July 14,2021

24. Chowkwanyun M, Reed AL. Racial health disparities and Covid19 - caution and context. N Engl J Med. 2020;383(3):201-3. https://doi.org/10.1056/NEJMp2012910. 
25. Webb Hooper M, Nápoles AM, Pérez-Stable EJ. COVID-19 and racial/ethnic disparities. JAMA. 2020;323(24):2466-7. https://doi. org/10.1001/jama.2020.8598.

26. Reitsma MB, Claypool AL, Vargo J, et al. Racial/ethnic disparities in COVID-19 exposure risk, testing, and cases at the subcounty level in California. Health Aff (Millwood). 2021;40(6):870-8. https://doi.org/10.1377/hlthaff.2021.00098.

27. Artiga S, Ndugga N, Pham O. Immigrant Access to COVID-19 Vaccines: Key Issues to Consider. Kaiser Family Foundation (KFF). Published January 13, 2021, 2021. https://www.kff.org/ racial-equity-and-health-policy/issue-brief/immigrant-access-tocovid-19-vaccines-key-issues-to-consider/. Accessed July 14

28. Webb Hooper M, Nápoles AM, Pérez-Stable EJ. No populations left behind vaccine hesitancy and equitable diffusion of effective COVID-19 vaccines. J Gen Intern Med. 2021. https://doi.org/10. 1007/s11606-021-06698-5.

29. Nguyen LH, Joshi AD, Drew DA, et al. Racial and ethnic differences in COVID-19 vaccine hesitancy and uptake. medRxiv. 2021. https://doi.org/10.1101/2021.02.25.21252402.

30. KFF COVID-19 Vaccine Monitor Dashboard. Kaiser Family Foundation. Published June 30, 2021. https://www.kff.org/coron avirus-covid-19/dashboard/kff-covid-19-vaccine-monitor-dashb oard/. Accessed July 14, 2021

31. Summers J. Little Difference In Vaccine Hesitancy Among White And Black Americans, Poll Finds, 2021. https://www.npr.org/secti ons/coronavirus-live-updates/2021/03/12/976172586/little-diffe rence-in-vaccine-hesitancy-among-white-and-black-americanspoll-find. Accessed July 14, 2021

32. Funk C, Tyson A. Growing Share of Americans Say They Plan To Get a COVID-19 Vaccine - or Already Have.; 2021. https://www. pewresearch.org/science/2021/03/05/growing-share-of-ameri cans-say-they-plan-to-get-a-covid-19-vaccine-or-already-have/. Accessed July 14, 2021

33. Hamel L, Lopes L, Kearney A, Sparks G, Stokes M, Brodie M. KFF COVID-19 Vaccine Monitor: June 2021. Kaiser Family Foundation (KFF). Published June 30, 2021. https://www.kff.org/ coronavirus-covid-19/poll-finding/kff-covid-19-vaccine-monitorjune-2021/. Accessed July 14, 2021

34. Loomba S, de Figueiredo A, Piatek SJ, de Graaf K, Larson HJ. Measuring the impact of COVID-19 vaccine misinformation on vaccination intent in the UK and USA. Nat Hum Behav. 2021;5(3):337-48. https://doi.org/10.1038/s41562-021-01056-1.

35. CDPH Allocation Guidelines for COVID-19 Vaccine During Phase 1A: Recommendations. California Department of Public Health. https://www.cdph.ca.gov/Programs/CID/DCDC/Pages/ COVID-19/CDPH-Allocation-Guidelines-for-COVID-19-Vacci ne-During-Phase-1A-Recommendations.aspx. Accessed July 15, 2021

36. Pfizer-BioNTech COVID-19 Vaccine. U.S. Food and Drug Administration (FDA). Published July 15, 2021. Accessed July 15, 2021. https://www.fda.gov/emergency-preparedness-and-response/coron avirus-disease-2019-covid-19/pfizer-biontech-covid-19-vaccine
37. Moderna COVID-19 Vaccine. U.S. Food and Drug Administration (FDA). Published July 7, 2021. Accessed July 15, 2021. https:// www.fda.gov/emergency-preparedness-and-response/coronavirusdisease-2019-covid-19/moderna-covid-19-vaccine

38. Governor Newsom Launches "Vaccinate All 58" Campaign based on Safety and Equity as First Vaccines Arrive to California. Office of Governor Gavin Newsom. Published December 14, 2020. Accessed July 15, 2021. https://www.gov.ca.gov/2020/12/ 14/governor-newsom-launches-vaccinate-all-58-campaign-basedon-safety-and-equity-as-first-vaccines-arrive-to-california/

39. Seniors 65+ Now Eligible to Receive COVID-19 Vaccine to Effectively and Efficiently Increase Vaccine Distribution, Reduce Hospitalizations and Save Lives. California Department of Public Health. Accessed July 15, 2021. https://www.cdph.ca.gov/Progr ams/OPA/Pages/NR21-015.aspx

40. Updated COVID-19 Vaccine Eligibility Guidelines. California Department of Public Health. Accessed July 15, 2021. https:// www.cdph.ca.gov/Programs/CID/DCDC/Pages/COVID-19/Vacci neAllocationGuidelines.aspx

41. Janssen COVID-19 Vaccine. U.S. Food and Drug Administration (FDA). Published July 13, 2021. Accessed June 15, 2021. https:// www.fda.gov/emergency-preparedness-and-response/coronavirusdisease-2019-covid-19/janssen-covid-19-vaccine

42. Joint CDC and FDA Statement on Johnson \& Johnson COVID-19 Vaccine. U.S. Food and Drug Administration (FDA). Published April 13, 2021. Accessed July 15, 2021. https://www.fda.gov/ news-events/press-announcements/joint-cdc-and-fda-statementjohnson-johnson-covid-19-vaccine

43. FDA and CDC Lift Recommended Pause on Johnson \& Johnson (Janssen) COVID-19 Vaccine Use Following Thorough Safety Review. U.S. Food and Drug Administration (FDA). Published April 26, 2021. Accessed July 15, 2021. https://www.fda.gov/ news-events/press-announcements/fda-and-cdc-lift-recom mended-pause-johnson-johnson-janssen-covid-19-vaccine-usefollowing-thorough

44. Provider Bulletin. California Department of Public Health. Accessed June 15, 2021. https://www.cdph.ca.gov/Programs/CID/ DCDC/Pages/COVID-19/Provider-Bulletin-2-12-21.aspx

45. Coronavirus (COVID-19) Update: FDA Authorizes Pfizer-BioNTech COVID-19 Vaccine for Emergency Use in Adolescents in Another Important Action in Fight Against Pandemic. U.S. Food and Drug Administration (FDA). Published May 13, 2021. Accessed July 15, 2021. https://www.fda.gov/news-events/pressannouncements/coronavirus-covid-19-update-fda-authorizespfizer-biontech-covid-19-vaccine-emergency-use

Publisher's Note Springer Nature remains neutral with regard to jurisdictional claims in published maps and institutional affiliations.

\section{Authors and Affiliations}

\section{Alexander Aram Bruckhaus ${ }^{1}$ - Aidin Abedi ${ }^{2} \cdot$ Sana Salehi ${ }^{1} \cdot$ Trevor A. Pickering $^{3} \cdot$ Yujia Zhang $^{1} \cdot$ Aubrey Martinez $^{1}$. Matthew Lai ${ }^{1} \cdot$ Rachael Garner ${ }^{1}$. Dominique Duncan ${ }^{1}$}

Alexander Aram Bruckhaus

bruckhau@usc.edu

Aidin Abedi

dr.aidin.abedi@gmail.com
Trevor A. Pickering

tpickeri@usc.edu

Yujia Zhang

zhan834@usc.edu 
Aubrey Martinez

aubreylmartinez@gmail.com

Matthew Lai

joyheiml@usc.edu

Rachael Garner

rachael.garner@loni.usc.edu

Dominique Duncan

dominique.duncan@loni.usc.edu

1 Laboratory of Neuro Imaging, USC Stevens Neuroimaging and Informatics Institute, Keck School of Medicine of USC,
University of Southern California, 2025 Zonal Ave., Los Angeles, CA 90033, USA

2 Department of Neurological Surgery, Keck School of Medicine, University of Southern California, Los Angeles, CA 90033, USA

3 Department of Population and Public Health Sciences, Keck School of Medicine, University of Southern California, Los Angeles, CA 90033, USA 